\title{
Immobilization of the Erupting Molar in the Syrian Hamster.
}

\author{
JOHN M. GREGG \\ University of Michigan Sclool of Dentistry, Ann Arbor, Michigan
}

The eruption of teeth, like many growth processes, seems to be an orderly and patterned sequence of events. The speed and frequency with which this process occurs make it ideal for studies in development and growth. Yet the interplay of growing tissues that results in eruption, although important, is still a problem.

A number of theories have been proposed that attempt to explain the eruptive movements of teeth and the maturation of supporting tissues. The first, and perhaps most obvious, idea has considered simple root elongation an eruptive force, the root lengthening and propelling the crown occlusally as if it were on stilts. Clinical observations, however, do not support this theory. Massler and Schour ${ }^{1}$ have observed that teeth often erupt even though there has been a failure in root elongation. Furthermore, the distance traveled by some teeth (cuspids) is much greater than the total root length. Experimental evidence has also opposed the root-elongation theory. Gowgiel ${ }^{2,3}$ prevented root formation in monkeys with irradiation and found that eruption of the crowns proceeded in its normal course. Schour and Van Dyke ${ }^{4}$ removed the pituitary glands of white rats and observed that, even though eruption was markedly retarded, a root "elongation" did continue in the form of a hard-tissue proliferation at the apex.

As an innovation of the root-elongation theory, Sicher ${ }^{5}$ has proposed that pulpal proliferation or vascularity is a primary factor in eruption. He believed that the expansion of pulpal tissue pressing against the stable fundic bone and hammock ligament pushes the tooth from its socket. Hertzberg

\footnotetext{
Parts of this investigation were presented at the 41 st General Meeting of the IADR, 1963

This study was supported by a Public Health Service fellow ship awarded through the General Research Support Grant 1-GS-22.
}

Received for publication June 26, 196,3 . and Schour, ${ }^{6}$ however, have removed the pulps of albino rats and observed normal eruption. Investigations such as these have led to the conclusion that neither root elongation nor pulpal proliferation causes eruption.

Another general theory of eruption proposes that proliferation and vascularity of periapical connective tissues function in pushing the developing teeth occlusally. Massler and Schour ${ }^{1}$ have concluded that vascularity of periapical connective tissues is probably responsible for eruption. They cite the work of Leist ${ }^{7}$ and King, ${ }^{8}$ in which the sympathetic innervation was destroyed on one side of the jaw in pups, kittens, and guinea pigs, producing a vasodilation or increased "vascularity." They observed an increased rate of eruption on the side with increased vascularity. Bryer ${ }^{9}$ similarly has described a "tissue tension" derived from the blood supply within the pulp and periodontal tissues which is responsible for eruption in the rat incisor. Taylor and Butcher ${ }^{10}$ have found, however, that an alteration of blood flow to periapical tissues of white rats did not alter the eruption rate. Main ${ }^{11}$ also has found no correlation between blood pressure and eruption rates in the rat. $\mathrm{O}^{\prime}$ Brien, Bhasker, and Brodie ${ }^{12}$ have compared successive stages of bone development in a special strain of rats and concluded that the follicle begins eruptive movement in the cap or bell stage. They compared the growth and eruption processes to sutural proliferation, where the dental follicle and periodontal membrane proliferate like the sutural connective tissue of bone. This proliferative growth causes an occlusal push of the tooth follicle and is followed by a secondary development of bony structures. Further evidence that connective-tissue growth is a primary factor in eruption was provided by the observation that when connective-tissue 
proliferation was suppressed, as after hypophysectomy, eruption was retarded. ${ }^{4}$

The growth and development of the alveolar bones and the periodontal fibers have been related to the eruption of teeth. Brash ${ }^{13}$ has demonstrated with madder-fed pigs that during eruption there was extensive growth of the alveolar crests. As cited by Tomes, ${ }^{14}$ the connective-tissue fibers then act together with the growing bone to pull the teeth occlusally. However, in 1924 Landsberger $^{15}$ found that removal of the tooth germs from the maxillae of dogs led to an absence of the alveolar process as well as a shortened maxilla and incomplete descent of the floor of the nose. Furthermore, Massler and Schour ${ }^{1}$ agreed with Meyer ${ }^{16}$ that during eruption in humans the periodontal fibers

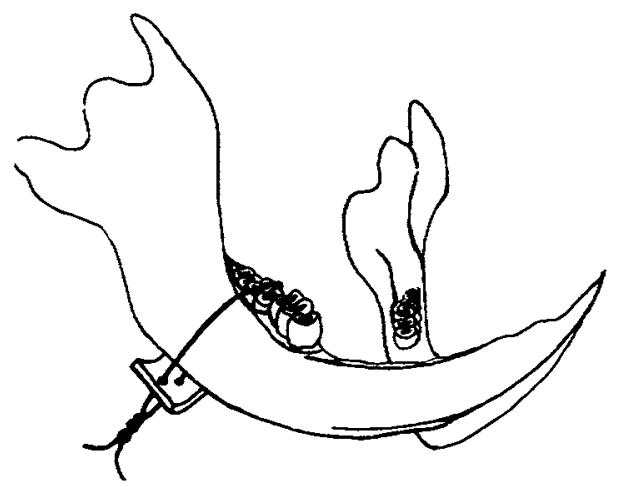

FIG. 1.-Diagram of hamster mandible with circumferential restricting wire in place over the second molar.

attached to the bone trabeculae angled upward to a higher point on the cementum and that the bone trabeculae themselves were oriented toward the direction of eruption. This evidence then led them to the conclusion that alveolar-bone growth occurs as a response to the eruption of teeth and that bone grow th will not proceed normally without eruption of the teeth.

The experimental approaches in studying the processes of eruption have been quite varied. Investigators have turned from simple microscopic observation to a controlled alteration of growth conditions in order to note the effects on eruption and dental morphology. There has been control of hormonal,17 vascular, ${ }^{1,11}$ and nervous ${ }^{18}$ factors as well as removal of certain structural elements, such as pulp ${ }^{6}$ and entire root. ${ }^{2,3}$ An approach to eruption study which has not been used to any extent involves a mechanical opposition to the eruptive forces. Clinically, teeth are often naturally restricted from erupting, as in tooth impaction. However, this is an uncontrolled situation and is rarely studied in terms of the consequences of restricted eruption itself. Taylor and Butcher, ${ }^{10}$ while studying rates of eruption in the white rat, touched on the idea of mechanically restricting eruption. One incisor was held from erupting by tension from a rubber band attached to a lower point on the adjacent incisor. They reported briefly that bone resorption as well as pulp death had occurred and that there was a folding of the root base and, after long periods of restriction, ankylosis.

It was the purpose of the present study (1) to develop a reproducible technique for restricting or immobilizing erupting molars in the Syrian hamster, (2) to apply the technique in a number of controlled situations, and (3) to analyze any structural changes which may have resulted from the immobilization in an attempt to gain information about the eruption of teeth and maturation of supporting tissues.

\section{Materials and Methods}

In a total of 52 Syrian hamsters, aged 14-30 days, a technique of circumferential wiring was employed as a means of immobilizing the molars which, in those young animals, were in the early stages of intraoral eruption.

Veterinary Sodium Nembutal, in a dosage of $0.3 \mathrm{mg} / 10 \mathrm{gm}$ of body weight, was found to be a safe and effective anesthetic. The dosage for each animal was divided, one-half being given intraperitoneally and one-half subcutaneously.

Although a completely aseptic technique was not practiced, the operating area was cleaned, all instruments were sterilized, and the external surgical area was shaved and swabbed with 70 per cent alcohol. A thumb forceps was positioned horizontally behind the incisors both to open the mouth and hold the tongue away from the field of operation. A curved needle threaded with 0.012 -gauge stainless-steel wire was introduced at the base of the mandible and passed through the floor of the mouth lin- 
gual to the molar. The needle then was arched over the molar, passed through the buccal mucosa, along the buccal aspect of the mandible, and through the skin. A small incision was made in the skin between the wires, and the wire ends were passed through holes in a thin, rectangular plate of stainlesssteel banding material. The plate then was positioned against the periosteum at the base of the mandible and the wires were twisted firmly against the plate (Fig. 1). The wound then was closed with skin clips.

Each hamster was given an intramuscular injection of Procaine Penicillin G, 50,000 units $10 \mathrm{gm}$ of body weight, to combat infection. The animals were finally returned to separate cages and maintained on an ad libitum diet of Purina Laboratory Chow, evaporated milk, and vitamin-supplemented water.

Seventy-one second or third molars were immobilized for varying lengths of time. In a few cases, the second molars were immobilized for a period of 1 week; the wires were then removed for 7 more days of free grow th before the animal was sacrificed. Other teeth were immobilized for varying periods of time ranging from 2 to 49 days before sacrifice. These time intervals were 2,4 , and 7 days and $2,3,4$, and 7 weeks.

The results were analyzed grossly, radiographically, and histologically. After sacrifice, vertical and horizontal radiographs were taken of bisected mandibles from representative animals. Gross dissection was performed in a few cases to analyze the extent of mandibular bone resorption. Other specimens were fixed in neutral 10 per cent Formalin, decalcified in 3 per cent hydrochloric acid, dehydrated in alcohol, and imbedded in paraffin. Sections then were cut at $10 \mu$ and stained with hematoxylin and eosin or Mallory's connective-tissue stain.

The following criteria were used to determine whether the wiring was "successful" and the teeth had in fact been immobilized: (1) wire visibly secure in place over the molar and around the mandible; (2) a visible crown-height difference compared to normally developed molars; and (3) no apparent infection, operative trauma, feeding problems, or any other factor resulting in illness or death.

\section{Results}

It was determined, by comparing the wired and unwired molars by gross dissection, radiographs, and histologic sections, that the surgical procedure of circumferential wiring is effective in preventing the active eruption of the developing molars of Syrian hamsters. The most significant problems, which usually resulted in death of the animal, were the development of infection, the necessity of early weaning, and traumatic effects caused by the anesthesia and the operation itself. Another problem was failure to position the wire precisely within the central fissure, which resulted in a tipping of the molar and the slipping of the wires into the embrasure. Because of these problems only 50 per cent of the total number of wired teeth was considered successful. However, as experience and proficiency increased, the rate of success increased to approximately 80 per cent in the later procedures, in which there was a minimum of operative trauma and antibiotic therapy was employed.

Histologic examination of molars restricted less than 3 days demonstrated no consistent morphologic disturbances. On the fourth day of restriction, however, definite changes appeared. The tips of the epithelial diaphragm were noticeably bent or pushed coronally. This distortion resulted in the epithelial diaphragm's being positioned either within the pulp chamber, partially outside the chamber, or partially outside and inside the chamber (Fig. 2). The position of the distortion of the epithelial diaphragm appeared to be a result of the angle at which the tooth attempted to erupt against the restricting wire. For example, when the tooth tipped mesially, as in Figure 2, the diaphragm was directed distally. As the molars were immobilized for longer periods, the root sheath continued to proliferate, and an accumulation of tissue resembling osteodentin appeared in this area (Fig. 3). After even longer periods of time, the apical osteodentin appeared as more dense, spherical bodies within the apical-root canal (Fig. 4).

Alveolar-bone growth continued in the interdental and interradicular areas even though eruption had been halted. This finding was supported by two observations: (1) the interradicular bone was found pressing 


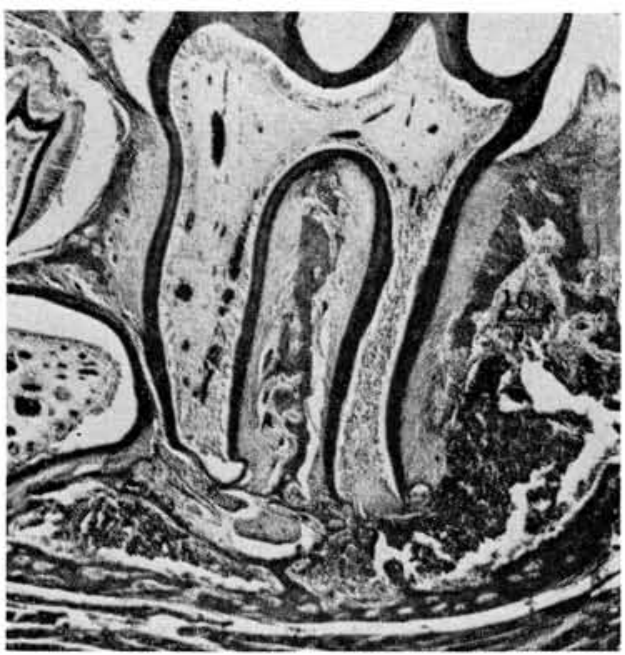

FIG. 2.-A hamster second molar which has been immobilized 4 days. Note the apical-root distortion and the involvement between interradicular bone and root bifurcation. (Mallory's connective-tissue stain.)

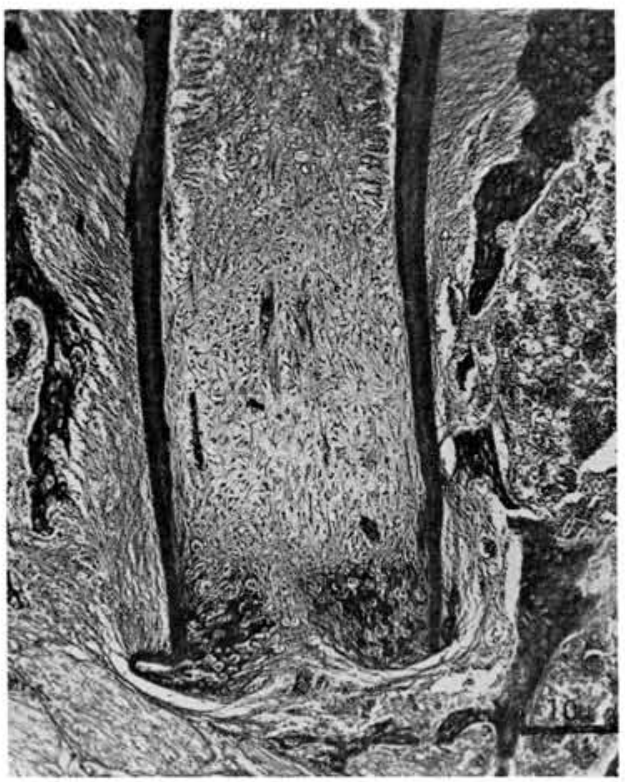

Fig. 3.-The apical-root of a second molar restricted 7 days. Note the accumulation of osteodentin, the sharp angulation of interradicular fibers $(l e f t)$, root resorption in this area, and the normal vascularity of pulp and periapical connective tissues. (Mallory's connective-tissue stain.)

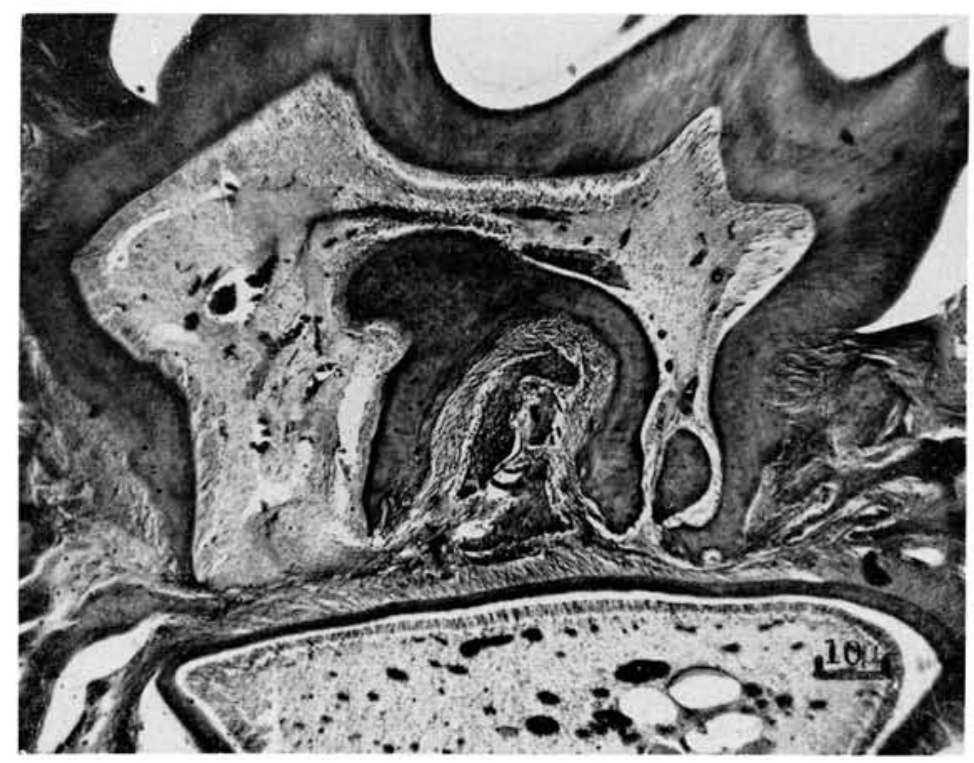

Fig. 4. - A third molar which has been immobilized for 2 weeks. Observe the very short, thick roots with little apparent lengthening. Also note the mass of hard tissue in the pulp and the suggestion of previous bifurcation involvement. (Mallory's connective-tissue stain.) 
against the root-bifurcation surface in those teeth immobilized 4 days, and root resorption was observed in these areas (Figs. 2 and 5), and (2) a comparison of the periodontal fiber angulation between immobilized and non-immobilized teeth showed that the fibers of the molars that had not been restricted were generally horizontal, whereas the fibers of molars that had been prevented from erupting were angled sharply upward to the bone (Fig. 5).

Although contact between interradicular bone and root surface was observed after 4 days of immobilization, an examination of molars restricted for more than 14 days showed a normal periodontal-membrane width in these areas (Figs. 4 and 6). Also, there appeared to be some suggestion of a previous involvement at the root-bifurcation surfaces in these longer immobilizations.

The immobilized molars showed a few changes within the pulp related to vascularity. A mild pulpal ischemia was seen in the coronal portions of a few teeth observed after short periods of immobilization. In a few teeth immobilized for long periods, there appeared to be an increased pulpal vascularity (Fig. 6), although this degree of vascularity also was seen in a number of control cases. The apical portion of the pulp chamber, however, showed no evidence of inflammatory response (such as hyperemia), other vascular changes, or edema. The periapical tissues of immobilized molars similarly showed no vascular changes.

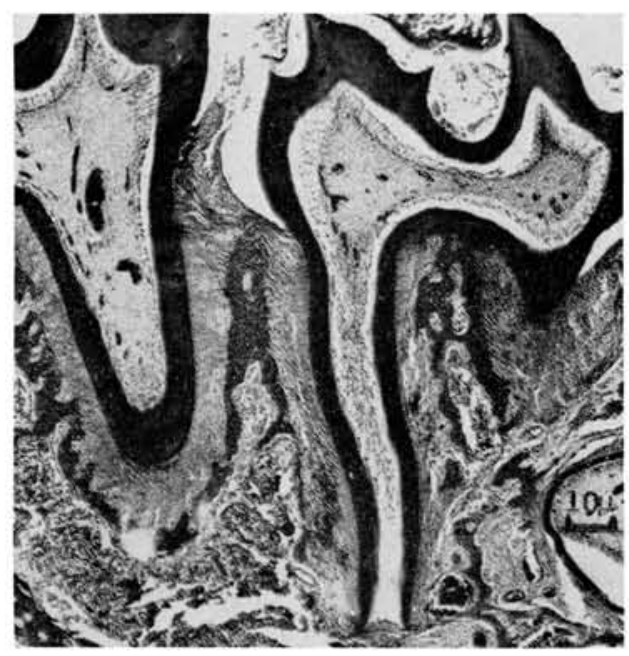

FIG. 5.-The second molar (right) has been restricted 4 days. Note the difference in angulation of the periodontal fibers of this tooth as compared to that of the fibers of the unrestricted first molar on the left. (Mallory's connective-tissue stain.)

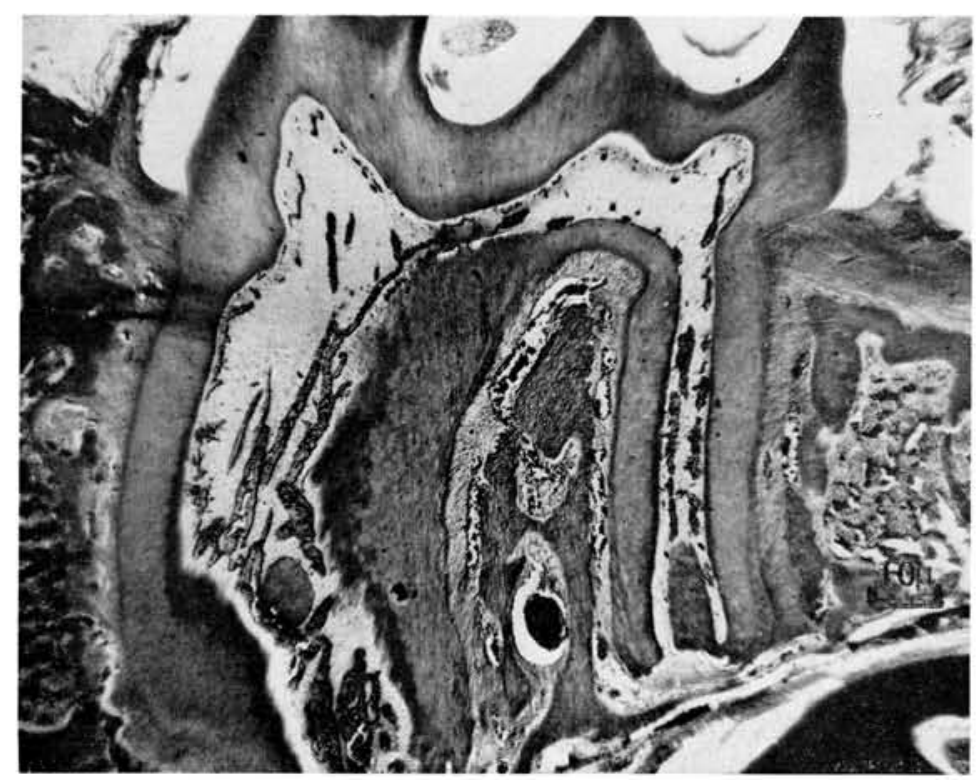

FIG. 6.-A second molar restricted for 7 weeks shows masses of osteodentin in the pulp as well as root resorption at the bifurcation and prominent pulpal vascularity. (Mallory's connective-tissue stain.) 
Different patterns of root development and change appeared, depending upon the age of the tooth when immobilization was initiated. When the molar was immobilized very early in the stages of root development, as in third molars, the root appeared to be very thick mesiodistally and very short or stubby along its length (Fig. 4). When root development had proceeded quite far before immobilization, the thickening was not ob-

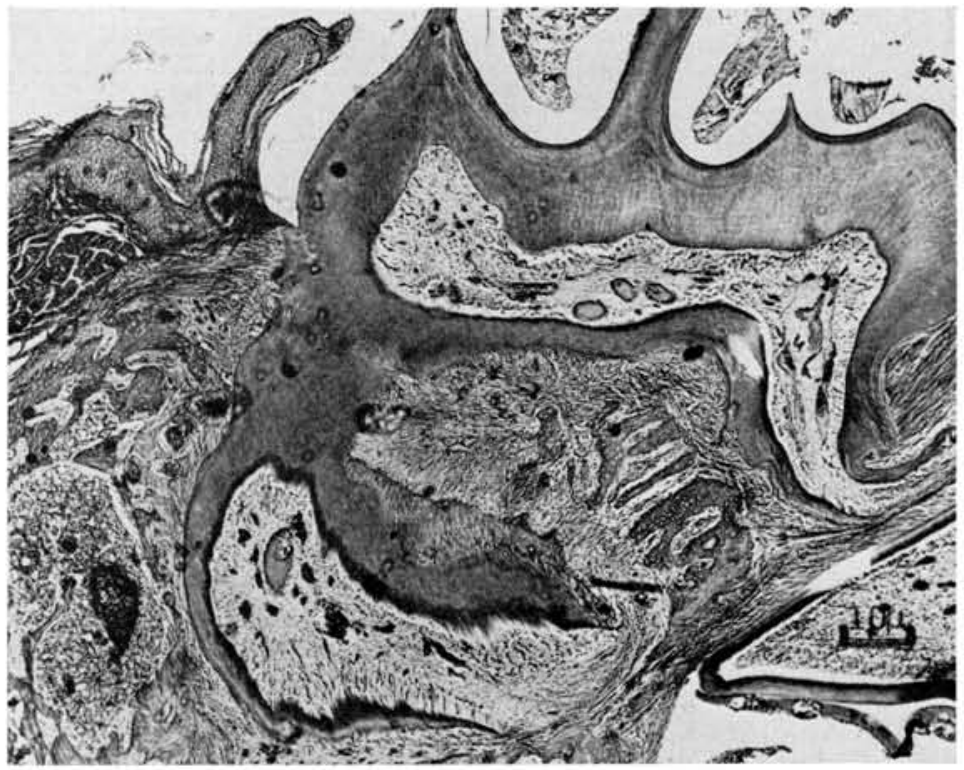

Fig. 7.-A third molar which has been immobilized for 3 weeks and has developed at an angle to the normal vertical axis. Note the long, curved distal root, the short mesial root, and the normal width of the periodontal membrane. (Mallory's connective-tissue stain.)

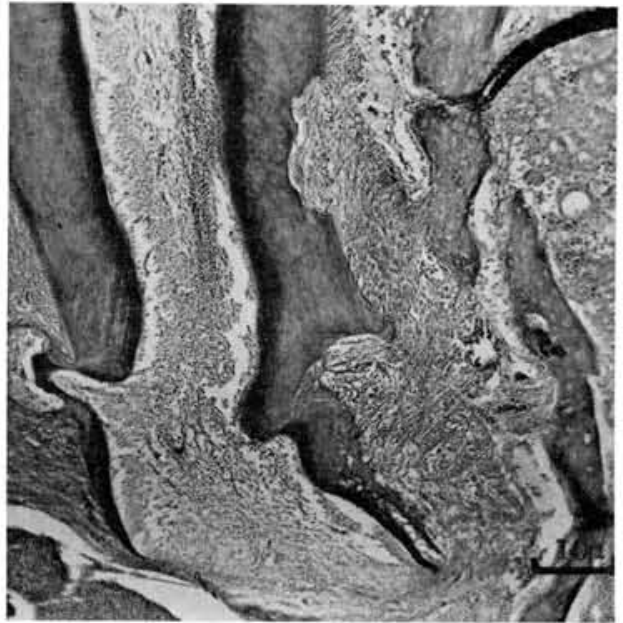

FIG. 8.-A second molar which has been restricted for 7 days and then released for 7 more days of free growth. Note the areas of root distortion and resorption. The apical spur of nearly normal root growth indicates development following release. (Mallory's connective-tissue stain.) served, nor was there evidence of a bending of the root portions that already had been formed. However, the apical distortion previously described was again seen (Fig. 3). When the molars were wired so that the eruptive force did not push against the wire in a completely axial vector, a difference be. tween mesial- and distal-root development appeared. In one case, the mesial root was short and the growth was stunted, while the distal root had grown posteriorly to a great length and width (Fig. 7).

In those cases in which the teeth were released from restraint after one week of immobilization, the molars quickly erupted into proper occlusion within 2 days. Histologically, the apical root showed evidence of bending, distortion, and resorption, but a spur of new, relatively straight root growth extending from the distorted area was observed (Fig. 8). In the released teeth the fiber attachment and orientation appeared normal, and the normal periodontal-membrane width had been re-established. 


\section{Discussion}

Restriction of eruption produces specific deviations from the normal grow th pattern of the root and supporting tissues. Analysis of these changes can be useful in developing ideas about the mechanisms of eruption and of the growth of supporting structures.

The types of changes seen at the root apex suggest that a simple elongation of root hard tissues is not a primary factor in tooth eruption. When hamster molars were prevented from erupting, the root apices showed no ability to lengthen, to develop a normal root form, or to contact and push against underlying bony structures. Evidence of this is seen in the case of a sharply angled molar where the roots vary greatly in length and form depending upon the restriction imposed by surrounding bone. In addition, when teeth were released from restriction and deformation, a spur of relatively normal root was seen to develop from the deformed area. It is suggested that the developing roots are quite plastic and the expression of their form seems to be dependent upon the immediate environment.

Pulpal growth or proliferation might be considered a part of over-all root growth and has, in itself, been described as a primary eruption force. ${ }^{5}$ There were no observations on immobilized molars, however, that would suggest that pulp tissues were attempting to proliferate. There was no cellular crowding or necrosis in the apical pulp, nor was there evidence of congestion, edema, or any change in degree of vascularity. Furthermore, the accumulations of osteodentin within the pulp chamber were in no instances pushed or extruded from the chamber by the proliferating pulp; rather, these masses were seen positioned even more coronally within the chambers.

The expansive and proliferative growth of periapical connective tissues might be considered a factor in eruption. There was no direct evidence gained from immobilized teeth that would suggest active growth by periapical connective tissues. There was no evidence of increased tissue density, crowding, or necrosis, which might be expected of confined, rapidly growing mesenchymal tissue. However, there was indirect evidence that periapical connective tissues could exert an occlusally directed force. With both root apex and underlying bone stabilized, the proliferating apical osteodentin appeared to be bent or distorted occlusally. This distortion appeared to be caused by no other force than pressure of connective tissue. Further observations suggested a connective-tissue force in the cases in which immobilized teeth were released from restraint and very quickly came into occlusion with restoration of normal periodontalmembrane width. These observations in immobilized teeth are inconclusive with regard to the importance and means of action of periapical connective tissues in eruption. It is probable, however, that proliferating connective tissue does exert an eruptive force through simple expansion.

Vascularity of both pulpal and periapical tissues has been proposed as a controlling factor in eruption., ${ }^{1,9}$ Some investigations have correlated increased vascularity with faster eruption, but contradictory evidence also has been presented. If, in fact, eruption is caused by the "tissue pressure" of pulpal and/or periapical tissues, then it might be expected that teeth restricted in their eruption would show evidence of vascular change in these areas. However, immobilized hamster molars showed no hyperemia, vascular proliferation, edema, or any other form of congestion in the apical or periapical tissues. Although nothing relative to the significance of vascularity in eruption may be concluded from these observations, there was no evidence to support the hypothesis that vascularity or vascular growth controls eruption.

Previous experimental evidence has suggested that alveolar-bone grow th is secondary to and dependent upon tooth eruption. ${ }^{1,15,16}$ The theory further implies that, if eruption were completely suppressed, the alveolar bone would fail to expand or to grow in an occlusal direction. Histologic observations of immobilized teeth tend to contradict this concept. First, interradicular-bone growth had continued after immobilization to make contact with the root bifurcation and eventually cause root resorption in this area. Second, periodontalmembrane width had been markedly reduced by bone expansion against the membrane. Additionally, when the molars were released, the quick eruption tended to restore normal membrane width, especially in the bifurcation areas. And, finally, in immobilized teeth the periodontal fibers were angled much more sharply toward the interdental bone 
than in normal teeth, indicating that the alveolar bone had continued to grow occlusally, carrying the fibers with it.

\section{Summary}

In an attempt to understand more fully the process of tooth eruption, hamster molars in the early stages of intraoral eruption were mechanically restricted from erupting by a surgical procedure of circumferential wiring. Seventy-one second and third molars were immobilized in this manner for periods of time ranging from 2 days to 7 weeks.

No histologic changes were noted in teeth immobilized for less than 3 days, but after 4 days the root tips appeared to be distorted and bent occlusally and interradicular bone was in contact with the root bifurcation. In addition, interdental alveolar bone seemed to have continued growing occlusally, carrying the attached periodontal fibers with it and causing a greater fiber angulation than in unrestricted teeth. After 1 week of immobilization, there was evidence at the apex of a disorganized proliferation resembling osteodentin. After longer periods of restriction, this tissue appeared as a dense mass in the apical-root canal and a normal periodontal-membrane width was observed. Molars released from their restriction resumed normal root growth, and the teeth came into occlusion in 1-2 days. At the apex, the pulp and surrounding connective tissues did not exhibit any necrosis or crowding due to contained cellular proliferation. In addition, there was no evidence of vascular changes within the apical pulp or periapical tissues.

From these observations the following conclusions were drawn: (1) Roots of immobilized teeth failed to lengthen properly and contact or push against underlying bony structures; therefore root elongation may be a result of the process of eruption rather than one of the primary causes. (2) Growth of alveolar bone, which seemed to continue even when eruption was restricted, may be an important factor in eruption by creating a stress on periodontal fibers. (3) No hyperemia, vascular proliferation, or edema was observed in the apical pulp or periodontal tissues; therefore, the findings of this study do not support the theory that vascularity or vascular pressure is a significant force in eruption.
I wish to thank Dr. James K. Avery, professor of dentistry, the University of Michigan School of Dentistry, for his guidance throughout the conduction, presentation, and writing of this study. Dr. Emmett R. Costich, professor of oral surgery, University of Kentucky College of Dentistry, was instrumental in the original planning. Further acknowledgment goes to Dr. Donald E. Knapp, assistant professor of dentistry, the University of Michigan School of Dentistry, for his practical suggestions and critical discussion and to Miss Elizabeth $W$. Haley for her technical assistance and editing of this paper.

\section{References}

1. Massler, M., and Schovr, I. Studies in Tooth Development: Theories of Eruption, Amer. J. Orthodont., 27:552-76, 1941.

2. Gowgies, J. M. Eruption of Irradiation-produced Rootless Teeth in Monkeys, $J$. dent. Res., 40:538-47, 1961.

3. 1961 (abstract).

4. Scholr, I., and VAN Dyke, H. B. Changes in the Teeth Following Hypophysectomy. I. Changes in the Incisor of the White Rat, Amer. I. Anal., 50:397$443,1932$.

5. Sicher, H. Tooth Eruption: Axial Movement of Teeth with Limited Growth, $J$. dent. Res, $21: 395-$ 402,1942 .

6. Hertzberg, F., and Schour, I. Effects of Removal of Pulp and Hertwig's Sheath on the Eruption of Incisors in the Albino Rat, $J$. dent. Res., 20:264, 1941 (abstract).

7. Lerst, M. Über den Einfluss des vegetativen Nervensystems auf die Zahne, Z. Stomat., 25:765-71, 1927.

8. KING, J. D. Dietary Deficiency, Nerve Lesions, and the Dental Tissues, $J$. Physiol. (Lond.), 88:62-7T, 1936.

9. Bryer, L. W. An Experimental Evaluation of the Physics of Tooth Eruption, Int. dent. J., 7:432-78, 1957.

10. Taylor, A. C., and Butcher, E. O. The Regulation of Eruption Rate in the Incisor Teeth of the White Rat, J.exp. Zoöl., 117:165-88, 1951.

11. MaIN, J. H. P. Relation between Unimpeded Erupted Rates and Blood Pressure in the Rat Incisor, J. dent. Res., 40:1276, 1961 (abstract).

12. O'Brien, C., Bhaskar, S., and Brodie, A. G. Eruption Mechanisms and Movement in the First Molar of the Rat, $J$. dent. Res., 37:467-84, 1958.

13. BRASH, J. C. Growth of the Alveolar Bone and Its Relation to the Movements of Teeth, Including Eruption, Internat. J. Orthodont., 14:196-223, 283$93,487-504,1928$.

14. Tomes, C. S. A Manual of Dental Anatomy, p. 225. 8th ed. New York: Macmillan, 1923.

15. LANDSBERgER, R. Histologic Research concerning the Growth of the Alveolar Process in Its Relation to the Development of the Tooth Germ, Denl. Cosmos, 66: $1334-45,1924$.

16. Meyer's Normal Histology and Histogenesis of the Human Teeth and Associated Parts, trans. and ed. H. R. Churchile, p. 241. Philadelphia: J. B. Lippincott and Co., 1935 .

17. Baume, L. V., Becks, H., and Evans, H. M. Hormonal Control of Tooth Eruption. I, II, III, $J$. dent. Res., 33: 80-114, 1954 .

18. Brown, G. N., Kupfer, S. R., and Darlington, C. G. Effects of Inferior Alveolar Nerve Severance on Eruption Rate of the Mandibular Incisor in the 10-Day-Old Wistar Albino Rat, Oral Surg., 14:122755,1961 . 